\title{
DifScal: A tool for analyzing difference ratings on an ordinal category scale
}

\author{
MARTIN C. BOSCHMAN \\ Technische Universiteit Eindhoven, Eindhoven, The Netherlands
}

\begin{abstract}
An algorithm for analyzing difference scaling results is described. Frequency data on ordered categories that represent perceived differences for a unidimensional psychological attribute are modeled according to Thurstone's judgment scaling model. The algorithm applies the gradient method for the maximum likelihood estimation of the model parameters. Two ways to calculate the start configuration for the model parameters are elaborated. The algorithm also provides asymptotic values for the standard errors of the estimates and three measures for the goodness of the model fit. An additional feature of DifScal is that it is suited to analyze incomplete data.
\end{abstract}

Whenever an experimenter wishes to measure a unidimensional perceptual attribute (e.g., brightness, loudness, perceived quality, etc.), he/she is faced with the problem that subjects are not able to directly report their (momentary) perceived attribute strengths for the presented stimuli. Experimental procedures are needed to allow subjects to convert the perceived relations between stimuli into responses. Psychometric assumptions for the response mechanism, such as Thurstone's law of comparative judgment (Thurstone, 1927), are required in order to analyse these responses. They specify how the subject's responses are related to the scale values (means) and dispersions (standard deviations) for stimuli on their internal psychological continuum.

Modeling of paired comparison results is well known for the case of a two-alternative forced-choice paradigm (e.g., Gulliksen, 1956; Mosteller, 1951a; Torgerson, 1958). In such a case, subjects must indicate, for each presented stimulus pair, which condition has the strongest attribute sensation (e.g., is brighter, louder, better, etc.). The subject's response space can be interpreted as a category scale with only two categories, and the measured frequencies can be modeled in order to derive the perceptual attribute strengths.

This article describes the algorithm underlying the program DifScal. This program has already been used extensively in the image quality research performed at our laboratory (Belaïd, 1999; Boschman \& Roufs, 1997; Martens \& Meesters, 1998; Meesters \& Martens, 1999). DifScal analyzes the results of paired comparison experiments in which perceived attribute differences for stimulus pairs are rated on a category scale. The number of categories

The author is indebted to Niek Versfeld for fruitful discussions on the subject, to Jean-Bernard Martens for the suggestions he offered, and to Huib de Ridder for giving permission to use his experimental data. Correspondence should be addressed to M. C. Boschman, IPO, Center for UserSystem Interaction, Technische Universiteit Eindhoven, P. O. Box 513, 5600 MB Eindhoven, The Netherlands (e-mail: m.c.boschman@tue.nl). is arbitrary. The categories can be labeled with adjectives or numbers. The attribute under investigation may be any unidimensional perceptual attribute. DifScal derives attribute values on an interval scale for the individual conditions in the stimulus set from the frequency data (number of times that each category has been selected for the presented stimulus pairs). The model underlying the DifScal analysis is the Thurstone scaling model. It is the same model that is also applied for the program ThurCatD (Boschman, 2000). The latter program analyzes direct ratings of attributes on a category scale.

In this paper, the Model section elaborates on the Thurstone scaling model underlying the DifScal algorithm. Starting from Thurstone's law of categorical judgment, the response model for categorical judgment of attribute differences is derived. In the two following sections, the constraints and parameters in DifScal are explained, and the likelihood function is derived from the model probabilities and the experimental data. The Estimation of Parameters section deals with estimation issues. First, the method of gradients, which is used to maximize the likelihood function, is described. Furthermore, "trivial" conditions (i.e., conditions that are always rated in extreme categories) and "empty" categories (i.e., unused categories) will be defined. They will be removed from the analysis whenever they occur. Also, two methods to calculate the start configuration of parameters are described. The first method is based on Gulliksen's least squares solution (Gulliksen, 1956) for incomplete paired comparison data. The second method, which is an alternative for cases in which Gulliksen's method is hampered because the data are too sparse, assumes that difference ratings can be interpreted to be linearly related to perceived differences. Finally, the criteria for stopping the iteration process are described. In the Estimation Errors and Model Fit section, a method to calculate an error measure (i.e., the Cramér-Rao bound for standard errors) is described briefly. This section also provides three measures for model fit. The first is a probability stress measure that in- 
dicates the deviation of the model probabilities from the experimental cell proportions. The second measure is a chi-square $\left(\chi^{2}\right)$ measure that is adapted from Mosteller's test statistic for two-interval paired comparison data (Mosteller, 1951b). The third measure is the well-known Wilks likelihood-ratio measure (Wilks, 1938), which is also asymptotically $\chi^{2}$ distributed. Finally, in the Sample Runs of DifScal section, a data set, taken from image quality research performed at our laboratory, is used to illustrate the functioning of the DifScal program. Special attention is given to the effect of reduction of number of filled cells in the data set. The DifScal results will also be compared with the results of the additive functional measurement model (Anderson, 1970).

\section{MODEL}

Thurstone (Thurstone, 1927; Torgerson, 1958) assumed that the strength of a psychological attribute for the stimuli in an experiment can be represented on a unidimensional psychological continuum. He therefore postulated the existence of a discriminal process that takes the physical stimuli as input and produces sensorial strengths on the psychologicalcontinuum for the attribute under study. Due to internal noise in this discriminal process, the output $x$ on the psychological continuum is assumed to be stochastic. It is likely that the discriminal process contains many independent noise sources that all contribute to the resultant noise. Then, according to the central limit theorem, a Gaussian distribution

$$
x_{i} \sim N\left(S_{i}, \sigma_{i}^{2}\right)
$$

is a plausible description of this stochastic output, although a logistic distribution is an often used alternative. The expected value of the distribution for stimulus $i$ is the scale value $S_{i}$; the standard deviation $\sigma_{i}$ is called the discriminal dispersion of the stimulus. Both scale value and dispersion may be different for different stimuli.

In order to understand the decision process under the paired comparison paradigm, we consider the distribu- tions of the outputs of the discriminal process for two stimuli, $i$ and $j$, with scale values $S_{i}$ and $S_{j}$ and dispersions $\sigma_{i}$ and $\sigma_{j}$, respectively. The subjects are asked to judge these stimuli and to indicate which has the largest magnitude with respect to the observed attribute. Thurstone assumed that the discriminal difference $d_{i j}=x_{i}-x_{j}$ is the internal variable that a subject applies to produce a response. Since the outputs of the discriminal process for both stimuli have normal distributions on an interval scale $\Psi$, the discriminal difference has a normal distribution on a ratio scale $\Psi^{\prime}$, which is an interval scale with an origin for zero differences. We obtain

$$
d_{i j} \sim N\left(S_{i}-S_{j}, \sigma_{i j}^{2}\right)
$$

with scale value $S_{i}-S_{j}$, and dispersion

$$
\sigma_{i j}=\sqrt{\sigma_{i}^{2}+\sigma_{j}^{2}-2 \rho_{i j} \sigma_{i} \sigma_{j}}
$$

where $\rho_{i j}$ is the correlation between the discriminal process outputs for stimuli $i$ and $j$. Hence, the probability that stimulus $i$ is judged to have a higher attribute value than stimulus $j$ equals

$$
P\left(x_{i}>x_{j}\right)=\Phi\left(\frac{S_{i}-S_{j}}{\sigma_{i j}}\right),
$$

where $\Phi$ denotes the cumulative standard normal distribution function. This is graphically depicted in Figure 1.

The above equations, which formulate Thurstone's law of comparative judgment, are used to construct the response model for the difference scaling paradigm. In difference scaling experiments, subjects are asked to rate their perceived attribute differences for pairs of presented stimuli on an ordered category scale. The $n_{c}$ categories may be labeled with numbers (e.g. $-3,-2,-1,0,1,2,3$ ) or adjectives (e.g., large preference for Condition 1, small preference for Condition 1, no preference, small preference for Condition 2, large preference for Condition 2) with the requirement that they have symmetrical semantics. In the case of an odd number of categories, the center category is used for neutral judgments (no preference or

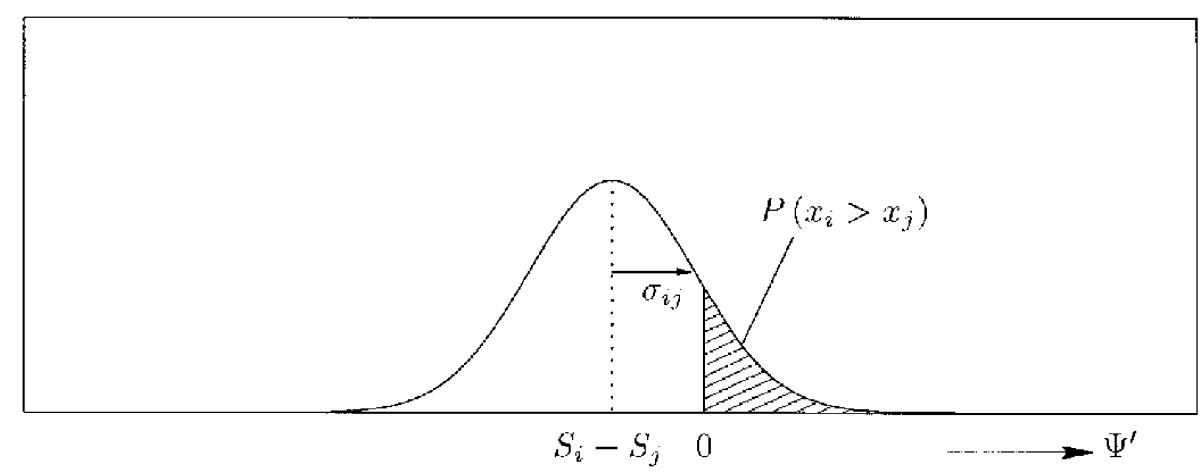

Figure 1. The magnitude of the perceived difference $d_{i j}$ between stimulus $i$ and stimulus $j$ is normally distributed on the ratio scale $\Psi^{\prime}$. For the depicted case, the scale value for stimulus $j$ is larger than the scale value for stimulus $i$. The shaded area corresponds to the probability that the subject reports stimulus $i$ to have a higher attribute magnitude than stimulus $j$. 
distinction). The special case of two categories is usually referred to as the two-alternativeforced-choice paradigm. It requires the subjects to indicate only which stimulus is preferred, in case of quality assessment, or the stimulus for which the attribute is perceived to be the strongest, in case of attribute scaling.

In the DifScal (difference scaling) algorithm, it is postulated that the aforementioned difference categories are represented by intervals on the ratio scale $\Psi^{\prime}$. It is postulated that the positions $b_{k}$ of the category boundaries also satisfy Gaussian distributions-that is,

$$
b_{k} \sim N\left(\beta_{k}, \delta_{k}^{2}\right) \text {. }
$$

The probability that the difference between the attribute values $x_{i}$ and $x_{j}$ of stimuli $i$ and $j$, respectively, is judged to be larger than category boundary $b_{k}$ equals

$$
P\left(x_{i}-x_{j}>b_{k}\right)=\Phi\left(\frac{S_{i}-S_{j}-\beta_{k}}{\sigma_{i j k}}\right),
$$

where the standard deviation

$$
\sigma_{i j k}=\sqrt{\sigma_{i}^{2}+\sigma_{j}^{2}+\delta_{k}^{2}-2 \rho_{i j} \sigma_{i} \sigma_{j}-2 r_{i k} \sigma_{i} \delta_{k}+2 r_{j k} \sigma_{j} \delta_{k}},
$$

depends on the discriminal dispersions $\sigma_{i}$ and $\delta_{k}$ of the stimuli and the category boundaries, respectively, on the correlations $\rho_{i j}$ between the attribute values for stimuli $i$ and $j$; and on the correlations $r_{i k}$ between the attribute value for stimulus $i$ and category boundary $k$. The derived probability $p_{i j k}$ that the difference between the attribute values of stimuli $i$ and $j$ is rated in category $k$ equals

$$
p_{i j k}=\Phi\left(\frac{S_{i}-S_{j}-\beta_{k}}{\sigma_{i j k}}\right)-\Phi\left(\frac{S_{i}-S_{j}-\beta_{k+1}}{\sigma_{i j, k+1}}\right) \text {. }
$$

\section{CONSTRAINTS AND PARAMETERS IN DIF SCAL}

In DifScal, it is assumed that the attribute values of all pairs of stimuli $(i, j)$ are uncorrelated-that is,

$$
\rho_{i j}=0 \text {. }
$$

It is also assumed that the dispersions for all conditions are equal, or

$$
\sigma_{i}=\sigma
$$

These assumptions are equal to those made in Case $\mathrm{V}$ of Thurstone's one-way classification (Thurstone, 1927) or Condition C of Torgerson's two-way classification (Torgerson, 1958) of cases of the law of comparative judgment. In DifScal, it is further assumed that subjects use fixed intervals on $\Psi^{\prime}$ corresponding with the difference categories-that is,

$$
\delta_{k}=0, \text { or } b_{k}=\beta_{k}
$$

for all $k$. The consequence of these constraints is that the standard deviation

$$
\sigma_{i j k}=\sigma \sqrt{2}
$$

is constant and, hence, independent of the stimulus pair $(i, j)$ and the category $k$. The category boundaries $\beta_{k}$ are assumed to be symmetrical around zero, in the sense that both categories $\left[-\beta_{m+1}^{\prime},-\beta_{m}^{\prime}\right)$ and $\left(\beta_{m}^{\prime}, \beta_{m+1}^{\prime}\right]$ exist on $\Psi^{\prime}$. If a neutral interval is included, it is represented by $\left[-\beta_{1}^{\prime}, \beta_{1}^{\prime}\right]$. With an even number of categories, the category bounds are

$$
-\infty,-\beta_{n_{b}}^{\prime}, \ldots,-\beta_{1}^{\prime}, \beta_{0}^{\prime}=0, \beta_{1}^{\prime}, \ldots, \beta_{n_{b}}^{\prime},+\infty,
$$

with $n_{b}=n_{c} / 2-1$ denoting the number of boundary parameters. Note that the halfway boundary $\beta_{0}^{\prime}$ is positioned at $\Psi^{\prime}=0$ and is therefore not a model parameter. With an odd number of categories, the category bounds are

$$
-\infty,-\beta_{n_{b}}^{\prime}, \ldots,-\beta_{1}^{\prime}, \beta_{1}^{\prime} \ldots, \beta_{n_{b}}^{\prime},+\infty,
$$

with $n_{b}=n_{c} \operatorname{div} 2$ boundary parameters. The interval boundaries are subject to the ordering constraint

$$
\beta_{k}<\beta_{l}
$$

for all $(k<l)$, implying that for the parameters

$$
\beta_{k}^{\prime}<\beta_{l}^{\prime}
$$

for all $(k<l)$, and

$$
\beta_{k}^{\prime}>0
$$

for all $\left(k=1, \ldots, n_{b}\right)$. Figure 2 depicts the response model after applying the above constraints.

Since the scale values on $\Psi$ are determined only up to an arbitrary linear transformation, DifScal sets the average of all scale values to zero-that is,

$$
\sum_{i}^{n_{s}} S_{i}=0
$$

The dispersion is used as the unit for the scale-that is,

$$
\sigma=1 \text {. }
$$

\section{LIKELIHOOD}

With the above model restrictions, the cell probabilities can be expressed as

$$
p_{i j k}=\Phi\left(\frac{S_{i}-S_{j}-\beta_{k}}{\sqrt{2}}\right)-\Phi\left(\frac{S_{i}-S_{j}-\beta_{k+1}}{\sqrt{2}}\right)
$$

The data can be summarized in a three-way contingency table, in which cell $(i, j, k)$ contains the number of times $f_{i j k}$ that stimulus pair $(i, j)$ is assigned to category $k$. If $n_{i j}$ is the total number of times that stimulus pair $(i, j)$ is presented, then the probability $P_{i j}$ of finding the distribution of frequencies over the categories $\left\{f_{i j k} ; k=1, \ldots, n_{c}\right\}$ for a stimulus pair $(i, j)$ is described by the multinomial distribution

$$
P_{i j}=n_{i j} ! \prod_{k=1}^{n_{c}} \frac{p_{i j k}^{f_{i j k}}}{f_{i j k} !}
$$




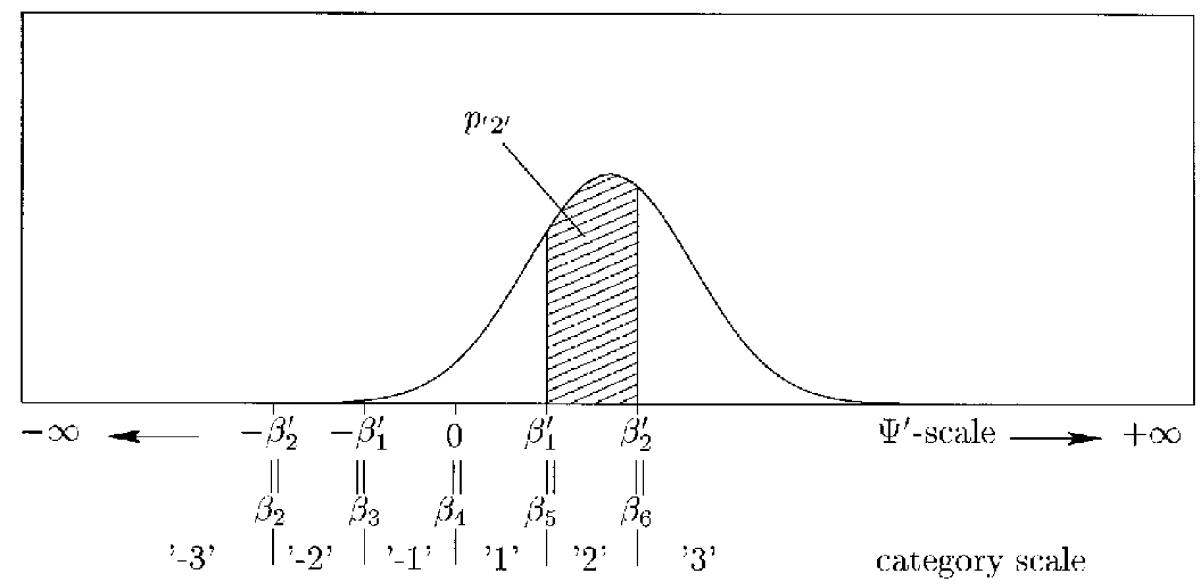

Figure 2. Relation between a numerical category response scale and the $\Psi^{\prime}$ scale for perceived attribute differences in the case of an even number of categories. The extreme intervals have one infinite boundary; in this example with six categories, $-3=\left(-\propto,-\beta_{2}^{\prime}\right]$, and $3=\left(\beta_{2}^{\prime}, \propto\right]$. The shaded area indicates the probability that the subject gives a rating in Category 2.

from which the log likelihood function

$$
\sum_{(i, j)}^{n_{f}}\left[\ln \left(n_{i j} !\right)+\sum_{k=1}^{n_{c}}\left(f_{i j k} \ln \left(p_{i j k}\right)-\ln \left(f_{i j k} !\right)\right)\right]
$$

is derived. Parameter estimation in DifScal is performed by maximizing the kernel of the log-likelihood function

$$
L=\sum_{(i, j)}^{n_{f}} \sum_{k=1}^{n_{c}} f_{i j k} \ln \left(p_{i j k}\right)
$$

by means of the method of gradients. The kernel of the log-likelihood function is the part of the likelihood function that depends on the model parameters. The algorithm allows data of experimental designs where not all stimulus pairs are presented. In Equations 22 and 23, the total number of cells for which $n_{i j}>0$ is denoted by $n_{f}$. Cells for which $n_{i j}=0$ are called structural zeros (Agresti, 1990).

\section{ESTIMATION OF PARAMETERS}

\section{Iteration With the Method of Gradients}

The parameter vector $\vec{\theta}$ is composed of the stimulus values $S_{i}$ and the boundary parameters $\beta_{k}^{\prime}$ :

$$
\vec{\theta}=\left[S_{1} \ldots S_{n_{s}} \beta_{1}^{\prime} \ldots \beta_{n b}^{\prime}\right]^{T} .
$$

DifScal applies the method of gradients to estimate the parameters. The log-likelihoodfunction is maximized by iterating $\vec{\theta}$ in the direction of the gradient of the loglikelihood $L$. More precisely, the parameter vector is iterated as follows:

$$
\vec{\theta}_{t+1}=\vec{\theta}_{t}+\alpha_{t} \frac{\nabla_{\vec{\theta}_{t}}}{\| \nabla_{\vec{\theta}_{t}}} \frac{\left(L_{t}\right)}{\left(L_{t}\right) \|},
$$

where

$$
\nabla_{\vec{\theta}}(L)=\sum_{(i, j)}^{n_{f}} \sum_{k=1}^{n_{c}} \frac{f_{i j k}}{p_{i j k}} \nabla_{\vec{\theta}}\left(p_{i j k}\right)
$$

denotes the gradient $L$ with respect to $\vec{\theta}$. The denominator of the right term of Equation 25 denotes the norm of the gradient vector, and $\alpha_{t}$ is the step size during iteration $t$. This step size depends on the progression of the iteration process and is determined according to the method proposed by Kruskal (Boschman, 2000; Kruskal, 1964).

\section{Trivial Stimulus Conditions and Empty Categories}

Before DifScal starts the iteration process, it checks the frequency table for trivial conditions and empty categories. A trivial condition $i$ is a condition that always scores in one of the two extreme categories when it is compared with other conditions:

$$
\sum_{j=1}^{n_{s}} f_{i j 1}=\sum_{j=1}^{n_{s}} n_{i j} \text { or } \sum_{j=1}^{n_{s}} f_{i j n_{c}}=\sum_{j=1}^{n_{s}} n_{i j} .
$$

Its attribute value hence is higher or lower than the attribute values of all other conditions. Consequently, the information on such a condition is insufficient, and leaving it in the analysis would hamper the iteration process to converge to maximum likelihood. It will hence be removed from the set before the iteration starts. Similarly, if a category $k$ and its opposite category $\left(n_{c}+1-k\right)$ are both found empty, that is, if

$$
\sum_{i j}^{n_{f}}\left(f_{i j k}+f_{i j\left(n_{c}+1-k\right)}\right)=0,
$$


then both categories will be removed before the iteration process starts.

\section{Start Configuration}

The iteration process requires some start configuration, which, in principle, could be any set of parameter values that complies with the aforementioned constraints. However, in order to prevent ending up with an estimation vector at a local maximum of $L$, it is important to start with a parameter vector that presumably lies close to the wanted configuration. In DifScal, one of two alternatives is used to calculate initial estimates for the parameters.

Gulliksen's least squares solution for incomplete data. The first method is an extension of Gulliksen's least squares solution for incomplete data that was originally proposed for two-interval data (Gulliksen, 1956; Torgerson, 1958). The method starts by deriving standard normal deviates

$$
z_{i j k}=\Phi^{-1}\left(P c_{i j k}\right)
$$

denoting the inverse standard normal distribution function for the cumulative proportions

$$
P c_{i j k}=\frac{1}{n_{i j}} \sum_{r=1}^{k-1} f_{i j r} .
$$

Since the model probability that stimulus $j$ has a higher attribute value than stimulus $i$ is equal to

$$
P\left(d_{i j}=x_{i}-x_{j}<0\right)=\Phi\left(\frac{S_{j}-S_{i}}{\sqrt{2}}\right),
$$

the standard normal deviate $z 0_{i j}$ that corresponds with $d_{i j}=0$ is an estimate for the normalized scale difference $\left(S_{j}-S_{i}\right) / \sqrt{2}$. In case of an even number of categories, $z 0_{i j}$ is equal to the deviate that represents the center boundary:

$$
z 0_{i j}=z_{i j \mu}
$$

where $\mu=\left(n_{c} \operatorname{div} 2+1\right)$. In case of an odd number of categories, $z 0_{i j}$ is equal to the average of the deviates corresponding with the boundaries of the neutral category:

$$
z 0_{i j}=\frac{1}{2}\left(z_{i j \mu}+z_{i j(\mu+1)}\right) .
$$

According to Gulliksen, the sum of squares

$$
Q=\sum_{(i, j)}^{n_{f}}\left[z 0_{i j}-\frac{\left(\hat{S}_{j}-\hat{S}_{i}\right)}{\sqrt{2}}\right]^{2}
$$

is minimized using matrix algebra, thus obtaining the initial scale values $\hat{S}_{i}\left(i=1 \ldots n_{s}\right)$. Cells of $z 0_{i j}$ with sample size $n_{i j}=0$ or with values $z 0_{i j}= \pm \infty$ are not taken into account. Since this minimization involves the calculation of the inverse on some auxiliary matrix (Torgerson, 1958), it may hamper with too sparse frequency data. In those cases, that auxiliary matrix will become singular. An alternative method (see next subsection) is applied to calculate the initial parameter values in case of a sparse set of frequencies.
The initial values for $\beta_{m}^{\prime}\left(m=1, \ldots, n_{b}\right)$ are derived from the initial values for $S_{i}$ and the standard normal deviates $z_{i j k}$. The model probability that the difference $d_{i j}$ is rated in a category lower than category $k$ is equal to

$$
P\left(d_{i j}=x_{i}-x_{j}<\beta_{k}\right)=\Phi\left(\frac{\beta_{k}-S_{j}+S_{i}}{\sqrt{2}}\right) .
$$

Hence, estimates for the boundary values $\beta_{k}$ can be derived:

$$
\hat{\beta}_{k}=\frac{1}{n_{f k}} \sum_{(i, j)}^{n_{f k}}\left[\sqrt{2} z_{i j k}+\hat{S}_{j}-\hat{S}_{i}\right],
$$

where $n_{f k}$ is equal to the number of cells for which $z_{i j k}$ has finite values and $n_{i j}>0$. Since DifScal applies the symmetry constraint for category boundaries, two estimates $\left(\hat{\beta}_{\mu+m}\right.$ and $-\hat{\beta}_{\mu-m}$ for an even $n_{c}$; or $\hat{\beta}_{\mu+1+m}$ and $-\hat{\beta}_{\mu-m}$ for an odd $n_{c}$ ) can be derived for the boundary parameters $\beta_{m}^{\prime}\left(m=1, \ldots, n_{b}\right)$. Dif Scal applies the weighted average of these estimates as initial value for the boundary parameters:

$$
\hat{\beta}_{m}^{\prime}=\frac{n_{f(\mu+m)} \hat{\beta}_{\mu+m}-n_{f(\mu-m)} \hat{\beta}_{\mu-m}}{n_{f(\mu+m)}+n_{f(\mu-m)}}
$$

for an even number of categories, and

$$
\hat{\beta}_{m}^{\prime}=\frac{n_{f(\mu+1+m)} \hat{\beta}_{\mu+1+m}-n_{f(\mu-m)} \hat{\beta}_{\mu-m}}{n_{f(\mu+1+m)}+n_{f(\mu-m)}}
$$

for an odd number of categories.

Alternative for sparse data. For particularly sparse frequency data sets, Gulliksen's method fails due to singularity of some auxiliary matrix (Torgerson, 1958). An alternative method is applied in those cases. This method assumes that the rank numbers of the difference categories are linearly related to the perceived attribute differences. First, arbitrary difference values are assigned to each category:

$$
v_{k}=k-\frac{n_{c}+1}{2}, \forall\left(k=1, \ldots, n_{c}\right) .
$$

Then, the expected unnormalized differences are calculated by

$$
\tilde{d}_{i j}=\frac{1}{n_{i j}} \sum_{k=1}^{n_{c}}\left(v_{k} \cdot f_{i j k}\right) .
$$

The pooled "within" standard deviation for these values is denoted by

$$
S D_{\tilde{d}}=\sqrt{\sum_{(i, j)}^{n_{f}}\left[\sum_{k}^{n_{c}}\left(f_{i j k} \cdot v_{k}^{2}\right)-\frac{1}{n_{i j}}\left(\sum_{k}^{n_{c}} f_{i j k} \cdot v_{k}\right)^{2}\right] \cdot\left[\sum_{i, j)}^{n_{f}}\left(n_{i j}-1\right)\right]^{-1}} .
$$

According to the model, differences have Gaussian distributions with noise spread $\sqrt{2}$, which is accomplished by defining normalized differences: 


$$
\hat{d}_{i j}=\frac{\sqrt{2}}{S D_{\beth}} \cdot \hat{d}_{i j}
$$

The initial stimulus values are calculated from these normalized differences. First $\hat{S}_{1}$ is arbitrarily set to 0 . Then, by iteration, for each known difference the other scale values are calculated from the already known initial values $\hat{S}_{k}$ :

$$
\hat{S}_{i}=\hat{S}_{k}+\hat{d}_{i k}, \text { or } \hat{S}_{j}=\hat{S}_{k}-\hat{d}_{k j} .
$$

Afterwards, the scale values are linearly transformed such that their average becomes equal to zero.

The start values for the category boundaries are obtained by

$$
\hat{\beta}_{m}^{\prime}=\frac{\sqrt{2}}{S D_{\tilde{d}}}\left(v_{(\mu+m)}-\frac{1}{2}\right)
$$

for $\left(m=1, \ldots, n_{b}\right)$.

\section{Stopping Criteria}

The DifScal algorithm applies two stopping criteria. The iteration is stopped if the number of iterations reaches a predefined maximum. The additional criterion is determined by the machine precision of the computations. The iteration is also stopped if the difference between current and previous value of the likelihood kernel $L$ is smaller than the machine precision.

\section{ESTIMATION ERRORS AND MODEL FIT}

DifScal calculates the pseudo-inverse of the Fisher matrix (e.g., van Trees, 1968) in order to find the asymptotic variance-covariance matrix for the estimated parameters. The asymptotic standard errors, which are also known as the Cramér-Rao bounds, are derived by taking the square root of the diagonal elements of the variance-covariance matrix. A detailed description of this operation can be found in Boschman (2000).

Three measures for the goodness of fit are derived from the deviation between model probabilities and experimental proportions. The probability stress, which equals the weighed average absolute deviation of model cumulative probabilities and observed cumulative proportions, is defined by

$$
P S=\frac{\sum_{(i, j)}^{n_{f}} \sum_{k}^{n_{c}}\left|f c_{i j k}-n_{i j} \cdot p c_{i j k}\right|}{n_{c} \sum_{(i, j)}^{n_{f}} n_{i j}},
$$

where $f c_{i j k}$ denotes the observed cumulative frequency and $p c_{i j k}$ denotes the cumulative probability for cell $(i, j, k)$. Small values for $P S$ indicate that the model predicts the experimental data properly. It is however hard to define a generalized criterion for the stress measure. From a number of Monte Carlo simulations, we found, however, an empirical rule of thumb for the stress criterion. It depends on the average cell sample size

$$
n_{a v}=\frac{1}{n_{f}} \sum_{(i, j)}^{n_{f}} n_{i j}
$$

According to this rule of thumb, it is assumed that the model fits the data properly if

$$
P S<\frac{0.13}{\sqrt{n_{a v}}} .
$$

The second measure is adapted from Mosteller's (Mosteller, 1951b) $\chi^{2}$ test statistic, which was meant for twointerval data. In DifScal, the test is adapted for multiple categories. This $\chi^{2}$ test indicates whether the set of model probabilities differ significantly from the observed proportions. The test works under the assumption that cell proportions are independent. This is a fair assumption if the number of categories is sufficiently large. An arcsine transformation is applied (Freeman \& Tukey, 1950) in order to also enable good approximations to a $\chi^{2}$ statistic for small sample sizes. The DifScal test statistic is

$$
\begin{aligned}
& \chi^{2}=\sum_{(i, j)}^{n_{f}} n_{i j} \sum_{k=1}^{n_{c}-1} \\
& \left(\arcsin \sqrt{\frac{f c_{i j k}}{n_{i j}+1}}+\arcsin \sqrt{\frac{f c_{i j k}+1}{n_{i j}+1}}-2 \arcsin \sqrt{p c_{i j k}}\right)^{2},
\end{aligned}
$$

where the number of degrees of freedom

$$
d f=n_{f}\left(n_{c}-1\right)-\left(n_{s}+n_{b}-1\right)
$$

is determined by the number of independent cells in the three-way contingency table, for which $n_{i j}>0$ minus the number of free model parameters.

The third measure is known as the Wilks likelihood ratio (Wilks, 1938). It is defined as

$$
G^{2}=2 \sum_{(i, j)}^{n_{f}} \sum_{k=1}^{n_{c}} f_{i j k} \ln \left(\frac{f_{i j k}}{n_{i j} p_{i j k}}\right) .
$$

It is asymptotically $\chi^{2}$ distributed with also $d f$ degrees of freedom. We found, in general, smaller values for the Wilks test statistic compared with the Mosteller $\chi^{2}$ with equal number of degrees of freedom (see, for example, the DifScal output in the Appendix). This indicates that the Mosteller test is more stern than the Wilks test.

The model fit for both $\chi^{2}$-based measures is assumed to be adequate if the upper-tail $\chi^{2}$ probability is sufficiently large (usually, larger than .05).

\section{SAMPLE RUNS OF DIFSCAL}

In this section, the DifScal analysis of a data set from de Ridder and Majoor (1990) is demonstrated. In their experiment, the stimulus set consisted of 11 different $\left(n_{s}=\right.$ 11) scale-space coded (Martens \& Majoor, 1989) digital images with natural content of which the bit rate was controlled by the quantization step $q$ that was varied from 1 to 127 . A value of $q=1$ resulted in an output image that was identical to the original image, whereas a value of $q=$ 127 resulted in a coded image that appeared consider- 
ably unsharp. With moderate values of $q$, another artifact denoted by "speckles" became visible. The relation between unsharpness and visibility of speckles on the one hand and image impairment on the other were studied. Each pair of images was simultaneously presented twice $\left(n_{i j}=2\right)$, and the perceived differences in overall impairment, visibility of speckles, and unsharpness were rated on a 21 -category $\left(n_{c}=21\right)$ numerical scale $(-10 \ldots$ $10)$. The results for the latter attribute (unsharpness) are used here as a sample data set in order to demonstrate the DifScal algorithm.

The subject responses are summarized in frequency files, of which an example is (partially) listed in the Appendix. It consists of $n_{c}$ lower triangular matrices in which the individual cells of matrix $k$ indicate how many times a stimulus pair $(i, j)$ is rated on category $k$. This frequency file was used as input for DifScal. The history information file and the output file that were produced during the DifScal run are also listed in the Appendix.

In order to demonstrate that DifScal can indeed deal with incomplete data, the original data set of 1 subject was reduced by removing the frequency data of randomly selected stimulus pairs. By doing so, the number of pairs $n f$ with $n_{i j}>0$ was reduced to $80 \%, 60 \%$, and $40 \%$ of the original number of pairs. In Figure 3, the results of the analyses of the reduced data sets are compared with those of the full data set. It shows that a reduction to $80 \%$ is hardly noticeable in the results. Although the deviation from the full data set results becomes larger for the $60 \%$ and $40 \%$ data sets, the linear correlation remains high $\left(80 \%, r^{2}=.995 ; 60 \%, r^{2}=.984 ; 40 \%, r^{2}=.988\right)$. The somewhat larger $r^{2}$ value for the $40 \%$ data set, rela-
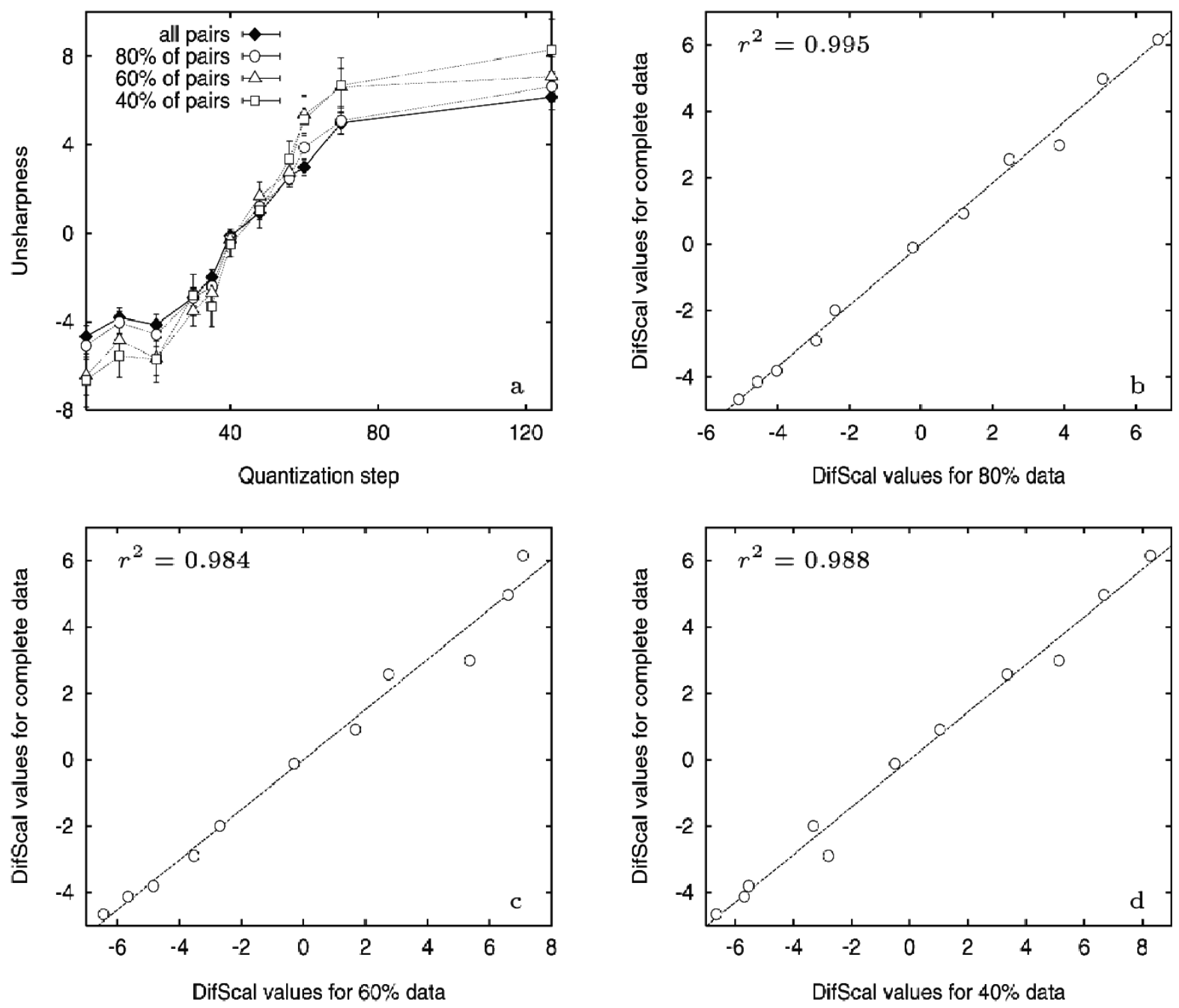

Figure 3. Panel a: Dif Scal results for the unsharpness scaling experiment described in the text. The independent variable was the quantization step of the scale-space coding algorithm. The figure depicts the results of 1 subject for the complete frequency matrix and three reduced data sets that were subsets of the original data. The vertical bars indicate twice the asymptotical standard errors of the estimated scale values. Panels b, $c$, and d depict the linear regression of the Dif Scal results for the complete data set with the results for the reduced data sets. 
tive to the value for the $60 \%$ set, can be explained by the stochastics of the random selection of the cells that were removed from the original set. In all cases, the Mosteller $\chi^{2}$ indicates that the model fits the data properly $(100 \%$, $\chi^{2}=728, d f=1,080 ; 80 \%, \chi^{2}=573, d f=860 ; 60 \%$, $\left.\chi^{2}=444, d f=640 ; 40 \%, \chi^{2}=266, d f=377\right)$. It may be counterintuitive that the stress value even decreases when the number of filled cells is decreased $(100 \%, P S=.048$; $80 \%, P S=.039 ; 60 \%, P S=.035 ; 40 \%, P S=.027)$. This can be explained, however, by the fact that less data need to be fitted on a model with an equal number of parameters. There is an increasing uncertainty in the parameter values for the incomplete data sets. In Figure 3, this is indicated by the increasing asymptotic standard errors for the more incomplete data sets.

Furthermore, the output data in the Appendix and Figure 3 show that the asymptotic standard errors of category boundaries and stimulus values first decrease and then increase with corresponding increasing values on the psychological continua. This phenomenon is not related to the properties of stimuli but is merely caused by a model artifact that can be explained intuitively. Since the extreme categories only have one finite boundary, it is likely that interval boundaries are less accurately determined for categories approaching these extreme categories. Consequently, scale values of stimuli will also be estimated with less accuracy if these categories are used when they are compared with other stimuli. This is the case for stimuli with scale values at the low or high end of the range.

An alternative, and simpler, model for interpreting subjects responses in a difference scaling experiment is the additive functional measurement (AFM) model (Anderson, 1970). This model assumes that numerical difference ratings can be interpreted as real numbers on the $\Psi^{\prime}$

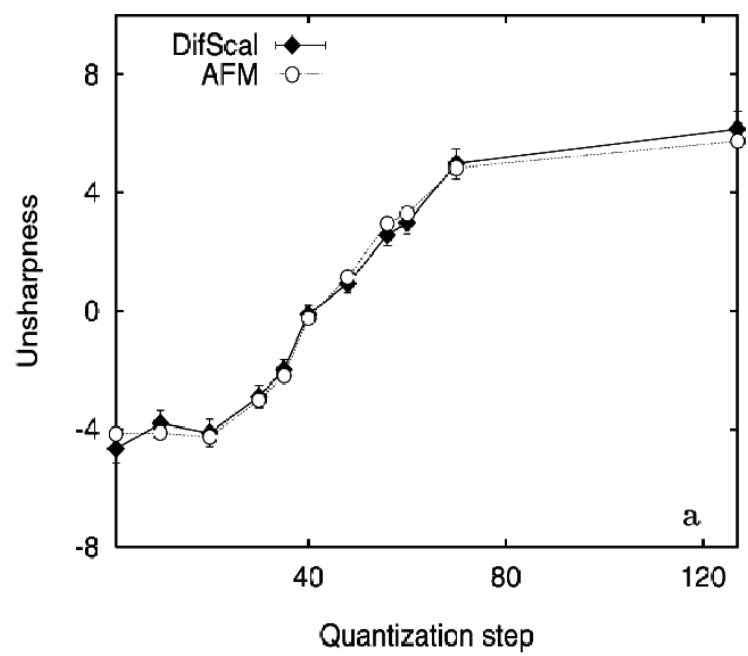

ratio scale. Hence, the response $\hat{d}_{i j}$ of a subject to a stimulus pair $(i, j)$ is assumed proportional to the difference of attribute strengths:

$$
\hat{d}_{i j} \propto d_{i j}=S_{i}-S_{j} .
$$

For a response matrix where data for all pairs $(i, j)$ are present,

$$
\hat{S}_{i}=\sum_{j=1}^{n_{s}} \frac{\hat{d}_{i j}-\hat{d}_{j i}}{2}
$$

provides an estimate for the attribute value $S_{i}$ of stimulus $i$. One should realize that these estimates are equivalent to the initial scale values for sparse data, as described in the subsection on the start configuration. The AFM model, however, needs a full matrix of rated differences in order to construct scale values. In Figure 4, the results of the DifScal and the AFM analyses are compared. In order to enable direct comparison between both models, the AFM data were linearly transformed such that the regression coefficient equals 1 . For the current data set of rated unsharpness differences, both models correlate quite linearly, implying that in this case the AFM model is adequate. Other statistical indices cannot be given, because the AFM model is deterministic, whereas DifScal deals with a stochastic response model. One disadvantage of AFM is that it needs a complete response matrix, which is not the case for the DifScal approach.

\section{Availability}

DifScal is an in-house program developed by the author. The Windows 95/98 version of DifScal is available for noncommercial use. It can be downloaded (from http:// www.ipo.tue.nl/homepages/mboschma/tools.htm).

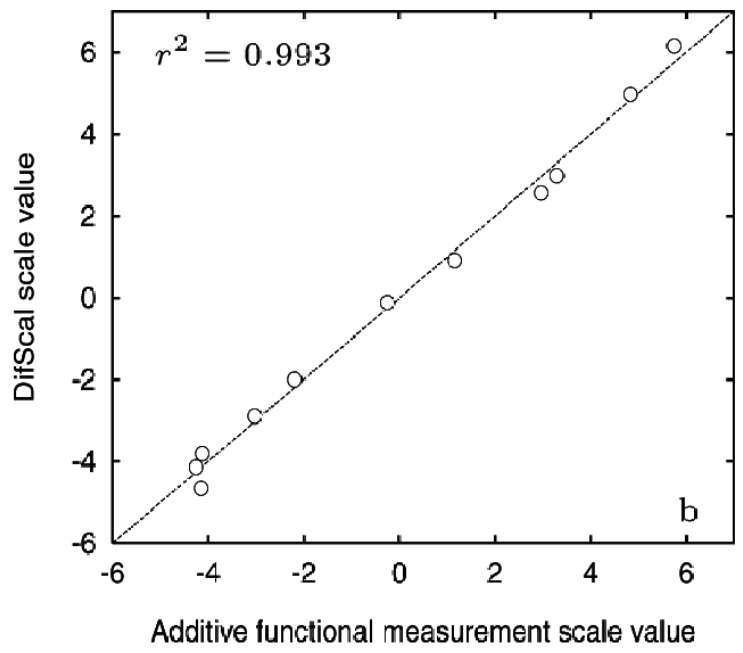

Figure 4. Panel a: The results of Dif Scal compared with those obtained by applying the additive functional measurement (AFM) model for the rated unsharpness differences. The AFM data were linearly transformed such that the regression coefficient $=1$. Panel b: The regression of the Dif Scal results with the transformed AFM results. The minor deviation from the regression line indicates the moderate nonlinearity of the functional measurement scale with respect to the psychological (Thurstone) scale. 


\section{REFERENCES}

Agresti, A. (1990). Categorical data analysis. New York: Wiley. Anderson, N. H. (1970). Functional measurement and psychological judgment. Psychological Review, 77, 153-170.

BELAïD, N. (1999). Perceptual linearization of soft-copy displays for achromatic images. Doctoral thesis, Eindhoven University of Technology.

Boschman, M. C. (2000). ThurCatD: A tool for analyzing ratings on an ordinal category scale. Behavior Research Methods, Instruments, \& Computers, 32, 379-388.

Boschman, M. C., \& Roufs, J. A. J. (1997). Text quality metrics for visual display units: II. An experimental survey. Displays, 18, 45-64.

DE Ridder, H., \& MAJoor, G. M. M. (1990). Numerical category scaling: An efficient method for assessing digital image coding impairments. In J. P. Allebach \& B. E. Rogowitz (Eds.), Human vision and electronic imaging: Models, methods and applications (Proceedings of the SPIE, Vol. 1249, pp. 65-77). Bellingham, WA: SPIE.

Freeman, M. F., \& Tukey, J. W. (1950). Transformations related to the angular and the square root. Annals of Mathematical Statistics, 21, 607-611.

GULLIKSEN, H. (1956). A least squares solution for paired comparisons with incomplete data. Psychometrika, 21, 125-134.

KrusKal, J. B. (1964). Nonmetric multidimensional scaling: A numerical method. Psychometrika, 29, 115-129.
Martens, J.-B., \& Majoor, G. M. M. (1989). The perceptual relevance of scale-space image coding. Signal Processing, 17, 353-364.

Martens, J.-B., \& Meesters, L. M. J. (1998). Image dissimilarity. Signal Processing, 70, 155-176.

Meesters, L. M. J., \& Martens, J.-B. (1999). Blockiness in JPEGcoded images. In B. E. Rogowitz \& T. N. Pappas (Eds.), Human vision and electronic imaging IV (Proceedings of the SPIE, Vol. 3644, pp. 245-257). Bellingham, WA: SPIE.

MostelLER,F. (1951a). Remarks on the method of paired comparisons: I. The least squares solution assuming equal standard deviations and equal correlations. Psychometrika, 16, 3-11.

MostelLER,F. (1951b). Remarks on the method of paired comparisons: III. A test of significance for paired comparisons when equal standard deviations and equal correlations are assumed. Psychometrika, 16, 207-218.

Thurstone, L. L. (1927). A law of comparative judgment. Psychological Review, 34, 273-286.

TORGERSON, W. S. (1958). Theory and methods of scaling. New York: Wiley.

van Trees, H. L. (1968). Detection, estimation and modulation theory: Part I. Detection and linear modulation theory. New York: Wiley.

WILKS, S. S.(1938). The large sample distribution of the likelihood ratio for testing composite hypotheses. Annals of Mathematical Statistics, 9, 60-62.

\section{APPENDIX Input and Output of DifScal}

In this Appendix, the DifScal analysis of a realistic experimental data set is demonstrated and its input and output are listed.

\section{The Frequency Input File}

This file describes the observed data to be analyzed. The first line gives the number of stimuli $n_{s}$ and the number of categories $n_{c}$. The rest of the file consists of $n_{c}$ lower triangle frequency matrices with cells (rowindex, columnindex) satisfying rowindex $\geq$ columnindex. If a subject responded to the instructed operation $d_{i j}=x_{i}-$ $x_{j}$ with a rating in category $k$ and $i \geq j$, then the frequency of cell $(i, j)$ of the kth triangular matrix was increased. If, on the other hand, $j>i$, then the rating was counted in cell $(j, i)$ of the $\left(n_{c}+1-k\right)$ th matrix. The latter is allowed, since we assumed that the categories have symmetrical semantics.

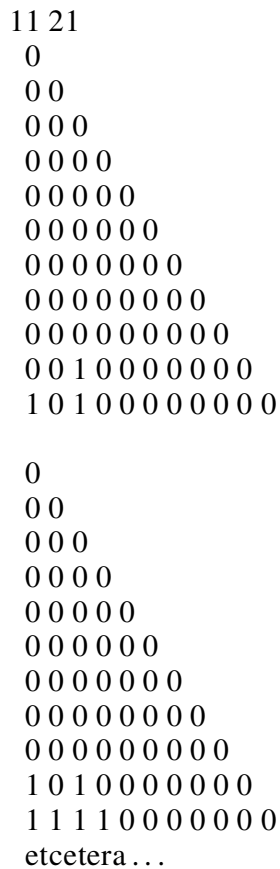




\section{APPENDIX (Continued)}

\section{History Output}

The following history information was displayed in a separate window during the run of the DifScal analysis of the frequency data listed before. It shows that the alternative way of calculating the start configuration was applied. Dif Scal needed 1,399 iterations to achieve its goal: a parameter configuration at maximum likelihood (the last likelihood deviation $<$ the machine precision).

History for C:IWINDOWS Desktopldsfileslu_hr.dsin generated by DifScal 2.2 on 8/30/00 at 15:45:33. DifScal 2.2 will analyse C:IWINDOWS $\backslash$ Desktopldsfileslu_hr.dsin using up to 5000 iterations.

$\mathrm{a} 0=0.0100, \mathrm{pmin}=0.0000010000$, extended output $=$ disabled, simple output $=$ disabled

DifScal read preference file C:IWINDOWS $\backslash$ Desktopldsfileslu_hr.dsin

Too sparse frequency table: Alternative startconfiguration is calculated. $\log$ likelihood kernel=-1.601 $162656350 \mathrm{E}+0002$ after 100 iterations. $\log$ likelihood kernel=-1.598946937106E+0002 after 200 iterations. $\log$ likelihood kernel $=-1.598548260177 \mathrm{E}+0002$ after 300 iterations. $\log$ likelihood kernel=-1.598476812621E+0002 after 400 iterations. log likelihood kernel $=-1.598463032066 \mathrm{E}+0002$ after 500 iterations. $\log$ likelihood kernel $=-1.598461749034 \mathrm{E}+0002$ after 600 iterations. $\log$ likelihood kernel=-1.598461542003E+0002 after 700 iterations. $\log$ likelihood kernel=-1.598461505068E+0002 after 800 iterations. $\log$ likelihood kernel $=-1.598461498952 \mathrm{E}+0002$ after 900 iterations $\log$ likelihood kernel=-1.598461497849E+0002 after 1000 iterations. $\log$ likelihood kernel=-1.598461497682E+0002 after 1100 iterations. $\log$ likelihood kernel=-1.598461497655E+0002 after 1200 iterations. $\log$ likelihood kernel=-1.598461497650E+0002 after 1300 iterations. Stop criterion reached (likelihood step <= precision) after 1399 iterations $\log$ likelihood kernel=-1.59846149764921E+0002

DifScal created results file C:IWINDOWS\Desktopldsfileslu_hr.dsout

DIFSCAL finished successfully!!

\section{DifScal Output}

The main output of the DifScal analysis for the sample input file is listed below. The output shows the estimated parameter values (i.e., stimulus values for unsharpness and interval bounds) and their asymptotic standard errors. Furthermore, the likelihood kernel value and the two measures for goodness of fit are listed. The latter indicate that the analysis was successful.

Output for C:IWINDOWS Desktopldsfileslu_hr.dsin generated by DifScal 2.2 on 8/30/00 at 15:45:33.

Configuration after 1399 iterations:

Estimated parameters (Noise spread parameter arbitrary set to 1):

Scale value parameters:

stimulus $1:$ scale value $=-4.6627 ;$ S estimate $=0.4976$

stimulus 2 : scale value $=-3.8100 ; \mathrm{S}$ estimate $=0.4428$

stimulus 3: scale value $=-4.1300 ; \mathrm{S}$ estimate $=0.4634$

stimulus 4: scale value $=-2.8985 ;$ S estimate $=0.3885$

stimulus 5: scale value $=-1.9891 ; \mathrm{S}$ estimate $=0.3457$

stimulus 6: scale value $=-0.1104 ; \mathrm{S}$ estimate $=0.3049$

stimulus 7: scale value $=0.9146 ;$ S estimate $=0.3144$

stimulus 8: scale value $=2.5634 ; \mathrm{S}$ estimate $=0.3722$

stimulus 9: scale value $=2.9880 ;$ S estimate $=0.3928$

stimulus 10: scale value $=4.9754 ; \mathrm{S}$ estimate $=0.5048$

stimulus 11: scale value $=6.1594 ;$ S estimate $=0.5799$

Interval bound parameters:

bound $1:$ value $=1.9627 ; \mathrm{S}$ estimate $=0.3020$

bound $2:$ value $=2.9775 ; \mathrm{S}$ estimate $=0.3455$

bound 3: value $=4.0082 ; \mathrm{S}$ estimate $=0.4049$

bound $4:$ value $=5.1342 ; \mathrm{S}$ estimate $=0.4807$

bound $5:$ value $=5.9482 ; \mathrm{S}$ estimate $=0.5359$

bound $6:$ value $=6.3156 ;$ S estimate $=0.5603$

bound $7:$ value $=6.6460 ; \mathrm{S}$ estimate $=0.5824$

bound $8:$ value $=7.2356 ;$ S estimate $=0.6227$

bound 9: value $=9.4037 ; \mathrm{S}$ estimate $=0.8024$

bound $10:$ value $=11.0603 ; \mathrm{S}$ estimate $=0.9465$ 
APPENDIX (Continued)

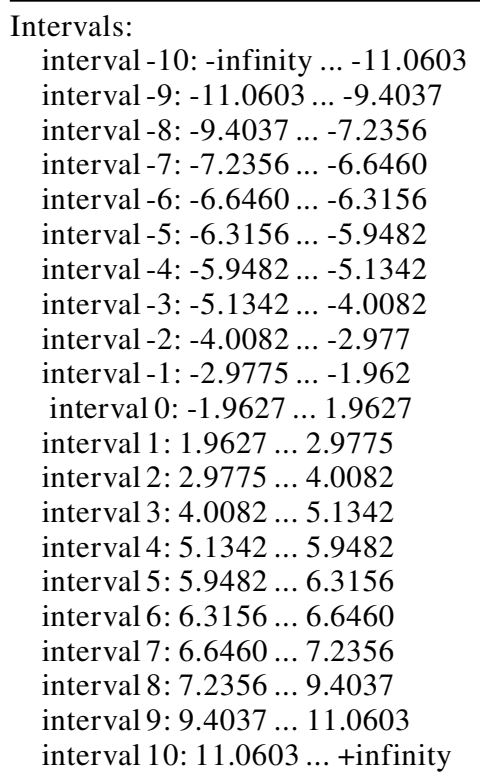

$\log$ likelihood kernel=-1.59846149764921E+0002

Model fit:

Probability stress $=0.047513->$ stress $<0.091924$

Model fit is OK according to rule of thumb for probability stress.

Mosteller Chi-square $=727.6398,1080$ degrees of freedom.

Upper tail P-value $=1.0000$ : model fit is OK: $(\mathrm{P}>0.05)$.

Wilks likelihood ratio G-square $=208.7888,1080$ degrees of freedom.

Upper tail P-value $=1.0000:$ model fit is OK: $(\mathrm{P}>0.05)$.

(Manuscript received November 9, 1999;

revision accepted for publication October 19, 2000.) 